\title{
Excimer laser ablation of microstructures: A numerical model
}

\author{
C. Paterson ${ }^{\mathrm{a})}$ \\ The Blackett Laboratory, Imperial College, London SW7 2BZ, United Kingdom
}

A. S. Holmes ${ }^{\text {b) }}$

Department of Electrical and Electronic Engineering, Imperial College, London SW7 2BT, United Kingdom

R. W. Smith

The Blackett Laboratory, Imperial College, London SW7 2BZ, United Kingdom

(Received 7 April 1999; accepted for publication 18 August 1999)

\begin{abstract}
A new model for the ablation of microstructures with excimer laser radiation is presented. The model is based on an interactive two-step approach. The local distribution of the light over the developing structure is evaluated for each pulse. This distribution is then used to calculate the local etch rate, and hence the change to the structure. Despite the assumptions inherent in the model, in particular assumptions made about the propagation of the light through a developing structure and about the etching behavior of the materials, results from the model agree with actual aspects of ablated structures. The model has been used with some success to predict the wall angles of trench structures ablated using partially coherent illumination from a fly's eye homogenizer. Predictions of the model show good agreement with the experiment results. In particular, the model correctly predicts the variation of the wall angles with incident fluence and also predicts structures with well defined wall angles over depths much greater than the depth of focus of the image. (C) 1999 American Institute of Physics. [S0021-8979(99)08522-9]
\end{abstract}

\section{INTRODUCTION}

Excimer laser ablation- the direct etching of material by pulsed ultraviolet light-has been the subject of sustained research activity since the early 1980s, when the technique was first demonstrated. ${ }^{1,2}$ The ablation process is characterized by the pulse-by-pulse removal of small amounts of material from the illuminated region of the target, with minimal damage to the surrounding area. The etch depth per pulse is typically in the range of a few hundred $\AA$ to a few $\mu \mathrm{m}$. This allows direct machining of patterns with resolutions down to the sub- $\mu \mathrm{m}$ range by mask projection techniques. ${ }^{3,4}$

The impetus for development of laser micromachining has come largely from the electronics industry, where excimer lasers have been used extensively for via drilling in chip packages and printed wiring boards. ${ }^{5,6}$ However, excimer lasers have also found applications across a much wider range of industries, covering a variety of materials from polymers to metals and ceramics. ${ }^{7}$ Their ability to etch biological materials without collateral damage has also led to a number of surgical applications. ${ }^{8,9}$ More recently, with the rapid expansion of research into microelectromechanical systems (MEMS), laser micromachining has been used for the direct manufacture of polymer microdevices, and for the production of molds from which metallic microstructures may be formed by replication. ${ }^{10-13}$

Considerable research effort has been devoted to understanding the fundamental physical and chemical processes involved in laser ablation, and numerous models have been proposed to explain the observed etching behavior of various

a)Electronic mail: carlp@ic.ac.uk

${ }^{b)}$ Electronic mail: a.holmes@ic.ac.uk polymer materials, in particular polyimide and poly-(methyl methacrylate). These models invariably assume the ablation process to be either photochemical, with the absorbed photons leading directly to bond breaking in the material, or photothermal, with the deposited energy being converted to heat, and subsequently leading to thermal decomposition; $;{ }^{14}$ it is generally accepted that in reality ablation proceeds by some combination of these two routes. The earliest models introduced the idea of a threshold fluence, below which material removal will not occur. ${ }^{15}$ Coupled with the assumption of linear absorption, this leads to the well-known "Beer's law" etching characteristic, which is obeyed by most materials over a limited range of fluences. ${ }^{16}$ Much of the later work has been devoted to explaining departures from this behavior by means of more sophisticated models which include such aspects as thermal diffusion and/or nonlinear absorption within the material, screening of the incoming radiation by the ablation plume, and incubation effects. ${ }^{17-23}$ Further insights into the ablation mechanism have been obtained by a variety of techniques, including spectroscopy of the ablation products and high speed photography. ${ }^{24}$

The modeling work to date has resulted in a comprehensive picture of laser ablation by a single illuminating beam at normal incidence. However, relatively little attention has been paid to the detailed form of microstructures formed by projection of a mask pattern. An important feature of polymer ablation by mask projection is the ability to machine with high accuracy features that are considerably deeper than the depth of focus of the imaging optics, and that have relatively high aspect ratios (i.e., ratios of machined depth to size of opening at the surface of the workpiece). The ability to produce vertical and even undercut sidewalls is also of great 


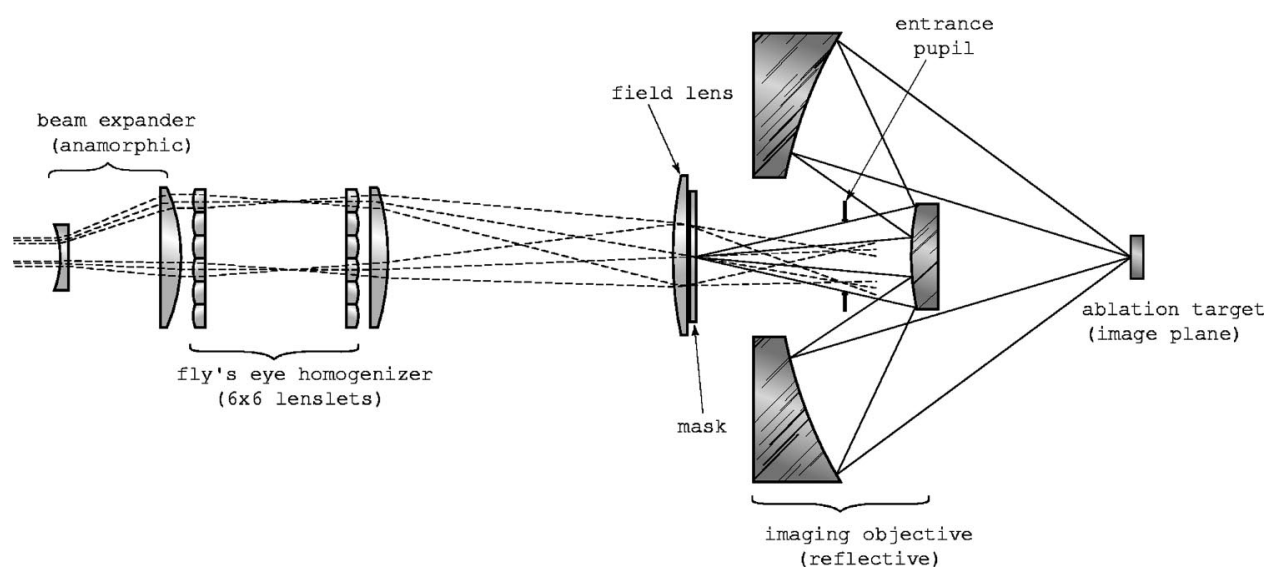

FIG. 1. Typical optical setup in a commercial laser micromachining workstation, comprising beam-forming optics, fly's eye homogenizer, mask and imaging lens.

practical interest. While these characteristics are well known, they have never been explained in terms of a consistent micromachining model. Perhaps more importantly, it has been impossible to predict in detail the shape of the threedimensional structure formed by projection of a given mask pattern; this information, which is particularly important in MEMS applications, has to be determined empirically for each new set of process conditions.

Attempts have been made to explain the variation of side-wall angle with fluence, based on a simple model described by Kahlert et al. ${ }^{4}$ According to this model, the sidewall angle obtained at any given laser fluence $F$ is determined by a critical angle of incidence, beyond which ablation cannot occur. The model further assumes that the material removal rate is a function only of the laser energy flux entering the surface, and that there is a definite ablation threshold $F_{T}$. With these assumptions, the side-wall angle $\Theta$ (measured from vertical) is expected to be given by the condition

$$
\alpha F \sin \Theta=F_{T},
$$

where $\alpha$ is a factor to account for reflection loss at the surface. The same model had been used earlier to explain the formation of conical structures during ablation of polyimide and poly(ethylene terephthalate) at low fluences. ${ }^{25}$ This model correctly predicts a steepening of the side-walls with increasing fluence. However, it cannot be expected to give quantitative information about the sidewall profiles obtained under typical micromachining conditions, because it takes no account of the illumination and imaging optics or the mask pattern. Furthermore, it is by no means clear that the central assumptions of the model are valid in general. For example, some materials do not exhibit a well-defined ablation threshold. $^{22}$

In this paper we present a numerical model for excimer laser micromachining by mask projection, based on pulseby-pulse propagation of the etched surface. For each laser pulse, we calculate the fluence distribution over the surface of the evolving structure, and then propagate the surface using an etch function which relates the local material removal rate to the fluence and angle of incidence. This kind of approach has been used previously by Dyer $e t$ al. to predict the surface profiles of convex and concave polymer surfaces after multipulse ablation under plane wave illumination, ${ }^{26}$ and more recently by Hodapp et al. ${ }^{27}$ to model ablation of holes in polyimide accounting for reflection effects.

Our model is able to make quantitative predictions about the side-wall profiles of laser-machined structures, taking into account all of the following: the mask pattern, the optical system (illumination and projection optics), the laser parameters (wavelength and fluence), the number of laser pulses, and the material etch characteristics. These predictions show good agreement with experimental results obtained using commercial excimer laser micromachining tools.

\section{MODELING TECHNIQUE}

The approach presented in this paper is to model the development of the machined structure as it is ablated pulse by pulse. It is assumed that the ablation events arising from successive pulses do not overlap in time. This is a valid assumption in most situations, since the interval between pulses (typically $\geqslant 10^{-2} \mathrm{~s}$ ) is usually far greater than the time scales involved in the ablation process itself (typically $\leqslant 10^{-6} \mathrm{~s}$ ). It is further assumed that the etching characteristics of the material remain stable throughout the micromachining process. This latter assumption, which has been adopted for simplicity, is not entirely valid with certain materials. For example PMMA is known to exhibit an incubation effect, where the ablation etch rate is affected by previous laser pulses.

The effect of each laser pulse is modeled in two stages. The first stage involves calculation of the radiation distribution incident on the surface of the partially formed structure. The second stage involves modeling the ablation and removal of material from the surface.

\section{A. The incident radiation}

The radiation incident on the surface of the partially machined structure depends both on the optical system used to form an aerial image in the vicinity of the structure, and on how the machined structure affects the propagation of the radiation in this aerial image. 
A typical optical setup is shown in Fig. 1. It consists of a mask containing the pattern to be ablated, an illumination system comprising a laser and beam homogenizer, and an imaging lens which forms the three-dimensional aerial image at the substrate. The illumination system performs two functions. The first is to reshape the excimer laser beam to give a more uniform intensity profile. The second function is to exploit the low spatial coherence of the excimer laser beam to produce partially coherent illumination of the mask, either from effectively an extended source, or from a set of discrete sources which are mutually incoherent.

The effect of this illumination will be taken into account in the modeling process. First however, the simple case of coherent illumination from a single point source will be considered before extending to partially coherent illumination.

\section{B. Coherent illumination}

If the mask is illuminated from a single monochromatic point source, then, assuming a perfect imaging system, the amplitude of the resulting wave in the image plane can be written approximately as

$$
\begin{aligned}
U_{0}(x, y) \propto & \tau(m x, m y) \exp [i(\alpha x+\beta y) k] \\
& \times \exp \left[\mathrm{i}\left(\frac{x^{2}+y^{2}}{2 l}\right) k\right] \otimes P(x, y),
\end{aligned}
$$

where $l$ is the distance from the point source to the image plane in the image space (which is usually the distance of the exit pupil from the image plane), $\tau(x, y)$ is the transmission function of the mask, $m$ is the magnification of the imaging lens and $\alpha$ and $\beta$ are the direction cosines of the point source from the image plane. $P(x, y)$ is the amplitude point spread function of the imaging system, i.e., the amplitude in the image that results from an on-axis point source and $k$ is $2 \pi / \lambda$ where $\lambda$ is the radiation wavelength.

Calculating how this wave propagates beyond the image plane and onto the surface of the partially machined structure is more complicated as the structure itself will affect the propagation by reflections, absorption and diffraction. A rigorous approach would have to take into account the full effect of the structure.

Existing modeling techniques for propagation of waves through complex media, such as the beam propagation method, ${ }^{28}$ rely on having relatively small, smooth variations in the refractive index of the media, but can cope with arbitrary refractive index functions within these constraints. Such techniques have been used extensively in modeling propagation in wave guides and integrated optics. Other techniques based on finding numerical solutions to Maxwell's equations, ${ }^{29}$ such as have been used in diffraction grating analysis, are more rigorous, but are computationally very costly and much less adaptable to arbitrary spatial functions of refractive index. Neither of these approaches is particularly suitable to the propagation of light through partially ablated structures that have very sharp, irregular variations of refractive index.

However, a closer look at the geometries of structures which are typical in excimer laser micromachining, and at the quantities of the radiation which are of importance in determining the ablation behavior, enables a number of approximations to be made which greatly simplify the modeling task. Typical structures tend to consist of surfaces which vary quite slowly with respect to the incident radiation wavelength, and which tend to face the incident radiation. At a point near the structure surface the radiation field consists of waves propagating towards the surface and waves propagating away from the surface. However, it can be assumed that it is only those waves propagating towards the surface (the incident waves) which are important in estimating the local ablation etch rate. Additionally, since the substrate materials being ablated have very high absorptivities at the laser wavelength, it can further be assumed that there is minimal propagation inside the substrate. We have further assumed that reflections from other parts of the surface may be neglected when calculating the incident field in any region. This assumption may not be valid under all conditions. However, we found that important features such as undercut sidewalls could be accounted for without reflections, at least for the material we investigated. Additionally, it is important that no parts of the surface lie in shadows cast by other features. This condition should be satisfied by most micromachined surfaces, which start off as plane substrates with no shaded regions. Such surfaces may develop regions which face away from the propagation direction of some or all of the radiation in the aerial image, but this can easily be accounted for.

Using these approximations, the wave at the surface of the structure can be calculated using free-space angular spectrum propagation. ${ }^{30}$ In fact, this is equivalent to calculating the wave as if the structure were not there at all (or, equivalently, as if it had a refractive index of one), and although for many other purposes the resulting errors would be unacceptable, they are concentrated in parts of the field which are relatively unimportant for modeling ablation. The amplitude of the wave at a point $P(x, y, z)$ on the surface is then given by

$$
\begin{aligned}
U(x, y, z)= & \iint A\left(k_{x}, k_{y} ; 0\right) \\
& \times \exp \left[\mathrm{i}\left(k_{x} x+k_{y} y+k_{z} z\right)\right] d k_{x} d k_{y},
\end{aligned}
$$

where $A\left(k_{x}, k_{y} ; 0\right)$ is the angular spectrum of plane waves in the plane $z=0$ (the top plane of the substrate). $A\left(k_{x}, k_{y} ; 0\right)$ is given by the Fourier transform of the amplitude at $z=0$

$$
\begin{aligned}
A\left(k_{x}, k_{y} ; 0\right)= & \left(\frac{1}{2 \pi}\right)^{2} \iint U_{0}(x, y) \\
& \times \exp \left[-\mathrm{i}\left(k_{x} x+k_{y} y\right)\right] d x d y,
\end{aligned}
$$

where $U_{0}(x, y)$ is given by Eq. (2). The Fourier transform pair of Eqs. (3) and (4) can be used to model the propagation of coherent illumination from the top plane of the substrate down to the surface of the developing structure.

\section{Partially coherent illumination}

The effect of the partially coherent illumination will now be considered. It is desirable that the illumination of the mask be not coherent in order to prevent coherent noise problems (e.g., speckle). This can be achieved easily with an 
excimer laser by employing the very low spatial coherence of its output beam. Typically there are about $10^{5}$ modes in the beam which will give a spatial coherence length of less than $10^{-2}$ times the beam width. For a typical beam width of $2 \mathrm{~cm}$, the spatial coherence distance is about $0.1 \mathrm{~mm}$ which, after expansion in the beam forming optics, is already greater than typical feature sizes on the mask. An homogenizer improves the uniformity of illumination by ensuring that each point in the mask plane receives radiation from many parts of the original laser beam profile. These illumination components are mutually incoherent and incident upon the mask from different directions.

A fly's-eye homogenizer ${ }^{31}$ achieves this by splitting up the laser profile using an array of lenslets (the fly's eye) to produce a set of discrete sourcelets, each of which illuminates the object from a different angle to the optical axis. The size of each sourcelet is sufficiently small that it can be considered as a quasimonochromatic point source (this is certainly the case for the sizes of the features which are of concern in the object).

The complex disturbance in the aerial image resulting from $N$ such sourcelets is given as

$$
V(x, y, z, t)=\sum_{i=1}^{N} V_{i}(x, y, z, t)=\sum_{i=1}^{N} u_{i}\left(t-\delta_{i}\right) U_{i}(x, y, z),
$$

where $u_{i}(t)$ is the complex amplitude of the disturbance of the $i$ th point source at time $t, \delta_{i}$ is the time delay between the point source and the origin of the image plane and $U_{i}(x, y, z)$ is the normalized spatial complex amplitude function for a monochromatic source located at the position of the $i$ th sourcelet, as given by Eq. (3). Substituting for $U_{i}$ from Eq. (3) gives

$$
\begin{aligned}
V(x, y, z, t)= & \sum_{i=1}^{N} u_{i}\left(t-\delta_{i}\right) \iint A_{i}\left(k_{x}, k_{y} ; 0\right) \\
& \times \exp \left[\mathrm{i}\left(k_{x} x+k_{y} y+k_{z} z\right)\right] d k_{x} d k_{y},
\end{aligned}
$$

where $A_{i}$ is the angular spectrum of plane waves for the $i$ th sourcelet as given by Eq. (4). Substituting Eq. (2) into Eq. (4) gives

$$
\begin{aligned}
A_{i}\left(k_{x}, k_{y} ; 0\right)= & \left(\frac{1}{2 \pi}\right)^{2} \iint \tau(m x, m y) \\
& \times \exp \left[\mathrm{i}\left(\frac{x^{2}+y^{2}}{2 l}\right) k\right] \exp \left\{-\mathrm{i}\left[\left(k_{x}-\alpha_{i} k\right) x\right.\right. \\
& \left.\left.+\left(k_{y}-\beta_{i} k\right) y\right]\right\} d x d y D\left(k_{x}, k_{y}\right) \\
= & A_{0}\left[\left(k_{x}-\alpha_{i} k\right),\left(k_{y}-\beta_{i} k\right) ; 0\right] D\left(k_{x}, k_{y}\right),
\end{aligned}
$$

where $A_{0}(x, y, z)$ is the aerial image resulting from a monochromatic, unit amplitude, on-axis point source (without the effect of the aperture) and $D\left(k_{x}, k_{y}\right)$ is the aperture of the system as a function of spatial frequency which is given by the Fourier transform of the amplitude point spread function
$P(x, y)$. This result reduces the computation required in evaluating the aerial image for partially coherent illumination.

The main quantities of interest in evaluating the ablation etch rate are the intensity and the energy flux vector. The intensity at a point $(x, y, z)$ in the aerial image is given by

$$
\begin{aligned}
I(x, y, z) & =\left\langle V^{*} V\right\rangle=\sum_{i=1}^{N} \sum_{j=1}^{N}\left\langle V_{i}^{*} V_{j}\right\rangle \\
& =\sum_{i=1}^{N} \sum_{j=1}^{N}\left\langle u_{i}^{*} u_{j}\right\rangle U_{i}^{*} U_{j} \\
& =\sum_{i=1}^{N} \sum_{j=1}^{N} \Gamma_{i j}\left(\delta_{i}-\delta_{j}\right) U_{i}^{*} U_{j},
\end{aligned}
$$

where $\Gamma_{i j}(\tau)$ is the mutual coherence of the point sources $i$ and $j .{ }^{32}$

Because the spacing between adjacent fly's eye lenslets is much larger than the spatial coherence of the beam, the mutual coherence between adjacent sourcelets is very small, and they can be considered as mutually incoherent, i.e.,

$$
\Gamma_{i j}(\tau)=\left\{\begin{array}{cc}
\left\langle\left|u_{i}\right|^{2}\right\rangle & i=j \\
0 & i \neq j
\end{array} .\right.
$$

Equation (8) then simplifies to

$$
I(x, y, z)=\sum_{i}^{N}\left\langle\left|u_{i}\right|^{2}\right\rangle\left|U_{i}(x, y, z)\right|^{2} .
$$

The energy flux vector is given by

$$
\mathbf{F}=\frac{1}{2 \mathrm{i} k}\left[\left\langle V^{*} \nabla V\right\rangle-\left\langle V \nabla V^{*}\right\rangle\right] .
$$

Substituting for $V$ from Eq. (5) gives

$$
\begin{aligned}
\mathbf{F} & =\frac{1}{2 \mathrm{i} k} \sum_{i=1}^{N} \sum_{j=1}^{N}\left\langle u_{i}^{*} u_{j}\right\rangle U_{i}^{*} \nabla U_{j}-\left\langle u_{i} u_{j}^{*}\right\rangle U_{i} \nabla U_{j}^{*} \\
& =\frac{1}{2 \mathrm{i} k} \sum_{i=1}^{N} \sum_{j=1}^{N} \Gamma_{i j}\left(\delta_{i}-\delta_{j}\right) U_{i}^{*} \nabla U_{j}-\Gamma_{j i}\left(\delta_{j}-\delta_{i}\right) U_{i} \nabla U_{j}^{*} .
\end{aligned}
$$

When the mutual coherence is as given in Eq. (9), the energy flux vector reduces to

$$
\mathbf{F}(x, y, z)=\frac{1}{2 \mathrm{i} k} \sum_{i=1}^{N} \mathbf{F}_{i}(x, y, z),
$$

where

$$
\begin{aligned}
\mathbf{F}_{i}(x, y, z)= & \left\langle\left|u_{i}\right|^{2}\right\rangle\left[U_{i}^{*}(x, y, z) \nabla U_{i}(x, y, z)\right. \\
& \left.-U_{i}(x, y, z) \nabla U_{i}^{*}(x, y, z)\right] .
\end{aligned}
$$

The aerial image can therefore be considered as an incoherent sum of the aerial images from each individual point source as given by Eq. (3). Figure 2 shows how the intensity of the aerial image changes beyond the focal plane for a long slit, $10 \mu \mathrm{m}$ wide at the image, which has been illuminated by a six-by-six fly's eye homogenizer of effective numerical aperture (NA) 0.18 . The effective NA in this context is de- 


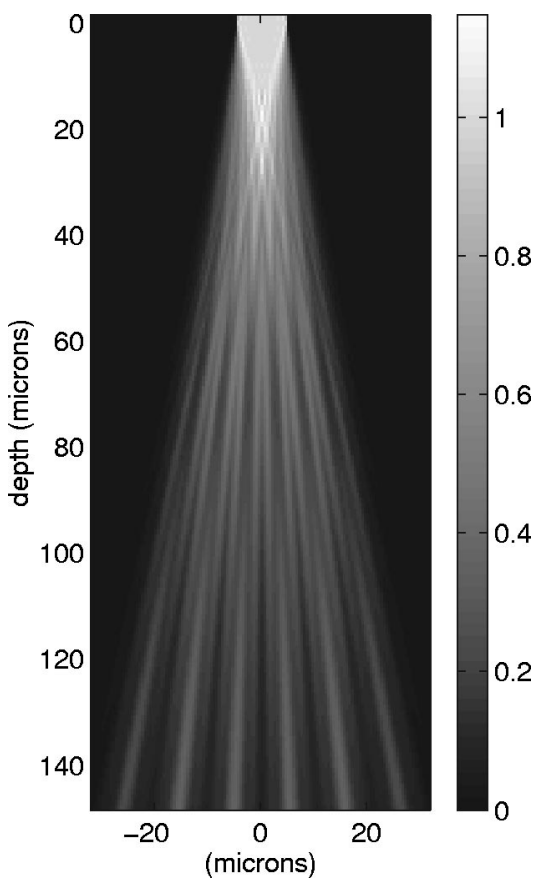

FIG. 2. Intensity distribution beyond the focal plane in the threedimensional aerial image of a long, $10 \mu \mathrm{m}$ wide slit. The picture shows a cross section through the aerial image, in a plane normal to the long axis of the slit.

fined as one half of the width (or height) of the square sourcelet array in the exit pupil divided by the focal distance. Note that, because the individual aerial images from the separate sourcelets propagate in slightly different directions, a banded intensity variation is produced away from focus. This behavior is supported by experimental observations. For example, Fig. 3 shows the patterns (viewed from above) produced by projecting a narrow slit onto plane polymer substrates positioned at distances of $0,50,100$ and $200 \mu \mathrm{m}$ beyond the focal plane. At $200 \mu \mathrm{m}$ the banding effect is clearly visible. Note that the bands are nonuniform in appearance, reflecting nonuniformity in the original laser beam profile. The two bands nearest the center are particularly weak because of the partial obscuration of the (reflecting) imaging lens used.

\section{The local etch rate}

In order to calculate the local etch rate per pulse over the surface of the partially machined structure, a detailed physical understanding of the ablation mechanism is desirable. This mechanism depends on the material parameters of the substrate as well as on the properties of the radiation incident upon it. Although there is a large body of published work on modeling the ablation of various materials, this has concentrated almost entirely on the case of uniform radiation incident normally upon the surface. There is very little information available on ablation behavior when the radiation is not at normal incidence, let alone when it consists of multiple incident waves. It is relatively easy to extend some of the simple physical models to include non-normal incidence. However our preliminary experimental results do not appear to support these extended models. As mentioned previously, (a)

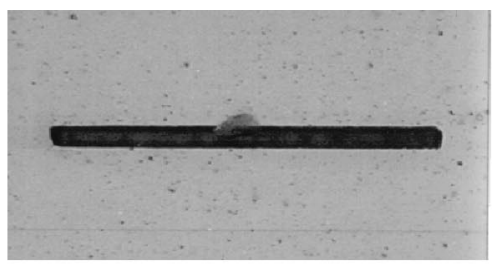

(b)

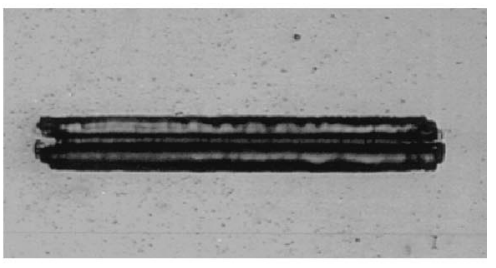

(c)

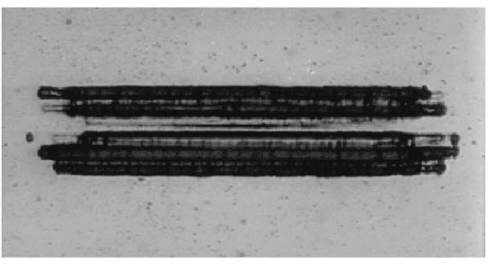

(d)

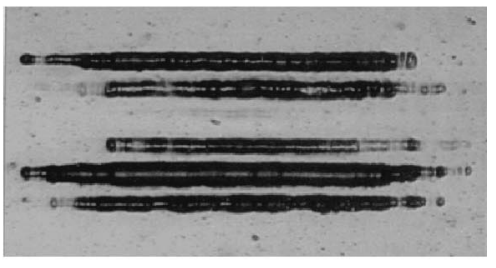

FIG. 3. Ablated patterns produced by projecting a $10 \mu \mathrm{m}$ wide slit onto plane polymer substrates positioned at distances of $0,50,100$, and $200 \mu \mathrm{m}$ beyond the focal plane, illustrating divergence of images from different sourcelets in the fly's eye: (a) $0 \mu \mathrm{m}$, (b) $50 \mu \mathrm{m}$, (c) $100 \mu \mathrm{m}$, (d) $200 \mu \mathrm{m}$.

there are many possible effects which may be causes of these discrepancies: heat conduction in the substrate, the shape of pulse energy envelope, plume shielding, etc. An attempt to account for these mechanisms is beyond the scope of this work at present.

Consequently, simple empirical models based on experimental observations and simple physical arguments have been assumed in order to estimate the local etch rate. In particular, the asymptotic model for the etch rate used by Kahlert et al. has been developed. It is assumed in this model that the local etch rate is a function of the total energy fluence (the flux due to the incident radiation integrated over the laser pulse). This determines the dependence of the etch rate on the angle of incidence. It is combined with the known etch rate behavior at normal incidence for the given material and laser properties to give an empirical etch rate model for non-normal incidence, so that the etch rate per pulse is given by

$$
\delta z=h\left(\sum_{i=0}^{N} f_{i}\right),
$$

where $f_{i}$ is the incident fluence at the surface which results from the $i$ th illumination sourcelet, and $h(E)$ is the etch rate 


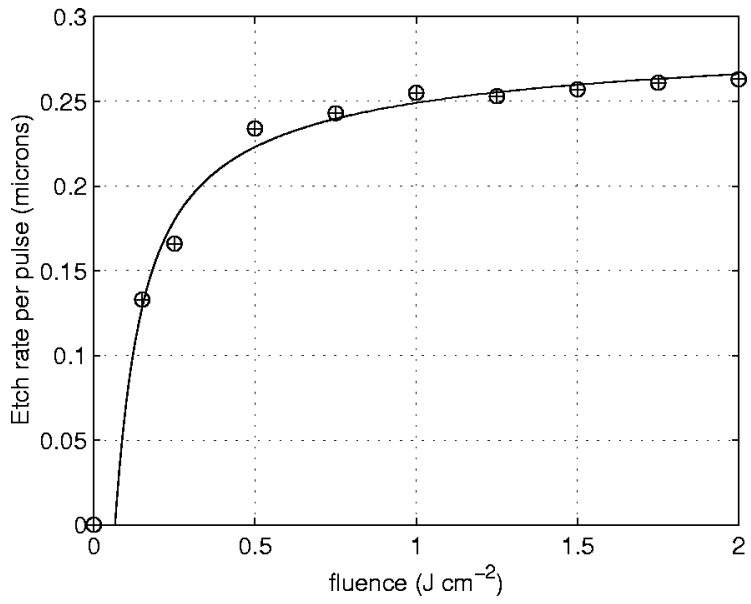

FIG. 4. Measured variation of etch depth per pulse with fluence for Du Pont RISTON (points), and a fitted empirical function given by Eq. (17) with $a$ $=0.82, f_{0}=0.066 \mathrm{~J} \mathrm{~cm}^{-2}, d=0.22 \mu \mathrm{m}$ (solid line).

for fluence $E$ at normal incidence, which can be an empirical function obtained by fitting experimental data for etch rates for uniform normal incidence illumination. The fluence due to the $i$ th sourcelet $f_{i}$ is given by

$$
\begin{aligned}
f_{i}= & \int_{\text {pulse }} \mathbf{F}_{i}(x, y, z) \cdot \hat{\mathbf{s}}(x, y, z) d t \\
= & \frac{1}{2 \mathrm{i} k} \int_{\text {pulse }}\left\langle\left|u_{i}\right|^{2}\right\rangle d t \times\left[U_{i}^{*}(x, y, z) \nabla U_{i}(x, y, z)\right. \\
& \left.-U_{i}(x, y, z) \nabla U_{i}^{*}(x, y, z)\right] \cdot \hat{\mathbf{s}}(x, y, z),
\end{aligned}
$$

where $\hat{\mathbf{s}}(x, y, z)$ is the local normal into the surface. In fact, when calculating the total fluence, only those components which contribute to the incident field (i.e., have positive flux across the surface) are included. The components with negative flux correspond to waves propagating away from the surface; such waves would in fact have already been absorbed higher up in the structure.

\section{E. Modeling procedure}

Using the derivations for the aerial image and the etching behavior described above, the structure propagation model has been developed. The angular spectrum of plane waves was discretized to enable discrete Fourier transform techniques and the fast Fourier transform algorithm to be used. The modeling procedure is as follows.

(1) Calculate the free-space aerial image which would be present in the region of the substrate if it were not there.

(2) Calculate the local etch rate $\delta z(s)$ over the current surface of the structure $\mathbf{S}(s)$ for one laser pulse using the empirical etch model and the aerial image. $[s$ is a single parameter uniquely describing the surface $\mathbf{S}(s)$ ].

(3) Calculate the new position of the surface defining the structure after removal of the material $\mathbf{S}^{\prime}(s)=\mathbf{S}(s)$ $+\delta z(s) \hat{\mathbf{s}}(s)$.

Steps (2) and (3) are repeated for the required number of pulses. The representation of the surface in the model and the
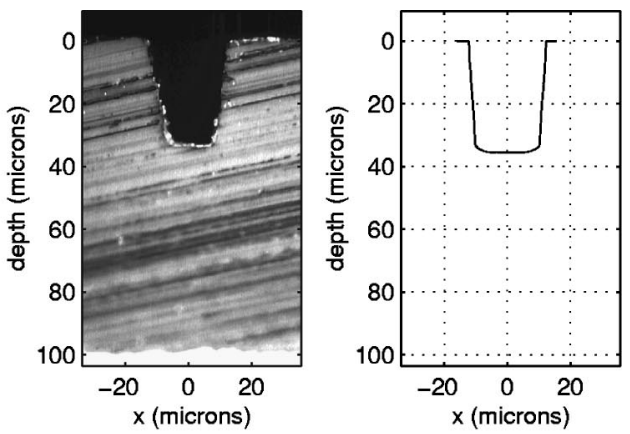

(a)
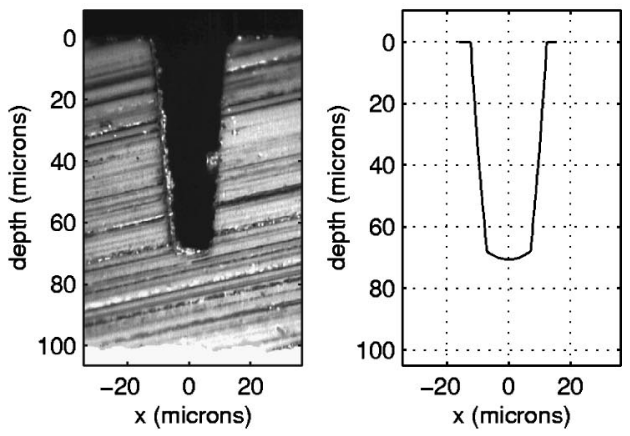

(b)
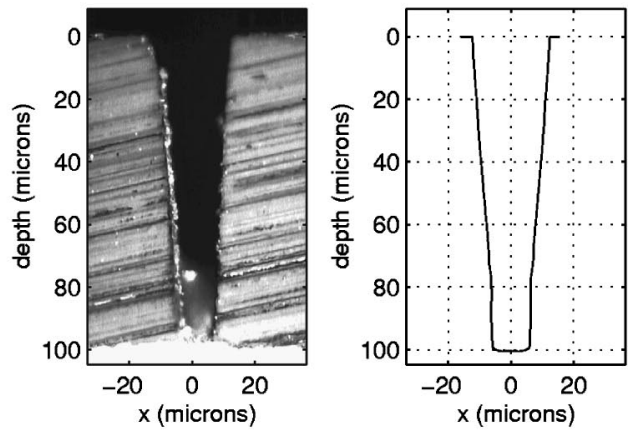

FIG. 5. Comparison between profiles predicted by our model (right) and actual ablated structures (left) for different numbers of pulses $N$ with an incidence fluence of $0.25 \mathrm{~J} \mathrm{~cm}^{-2}$ : (a) $E=0.25 \mathrm{~J} \mathrm{~cm}^{-2}, N=200$; (b) $E$ $=0.25 \mathrm{~J} \mathrm{~cm}^{-2}, N=400$; (c) $E=0.25 \mathrm{~J} \mathrm{~cm}^{-2}, N=600$.

mask functions are two dimensional (i.e., trenches, steps, etc.), however, the full three-dimensional illumination is used.

\section{RESULTS}

The structure propagation model described above was used to model the ablation of long trenches in a dry film photoresist material (Du Pont RISTON). Dry film resists, which were developed for the printed circuit board industry, are of interest for MEMS applications because they offer ease of application, good thickness uniformity, and film thicknesses up to several hundred $\mu \mathrm{m} .{ }^{33,34}$ Resist films of $100 \mu \mathrm{m}$ thickness were laminated onto 4 in. diam silicon wafers, and crosslinked prior to ablation by flood exposure in a UV contact printer.

Experiments were carried out using an Exitech Series 7000 laser micromachining workstation. This machine employs a Lambda Physik LPX 110i excimer laser, operating at 

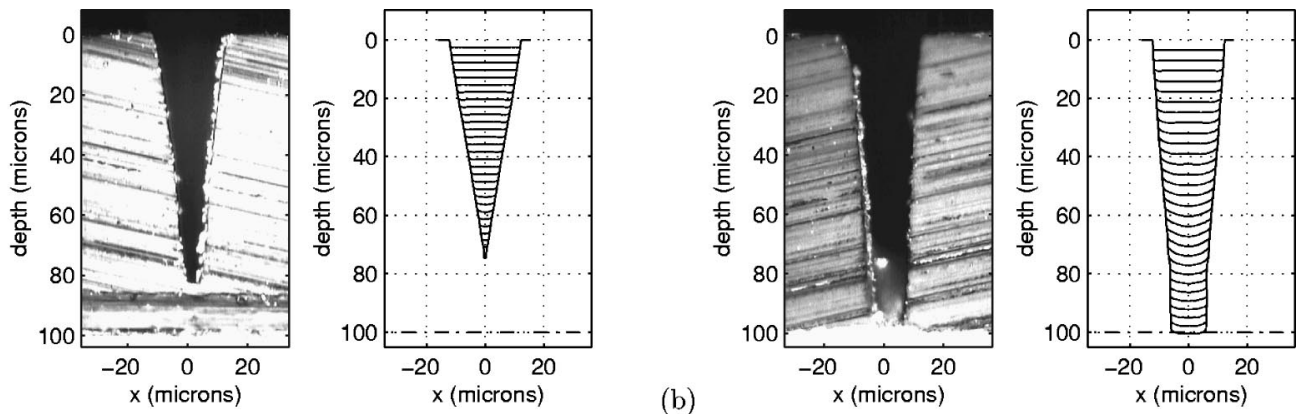

(a)

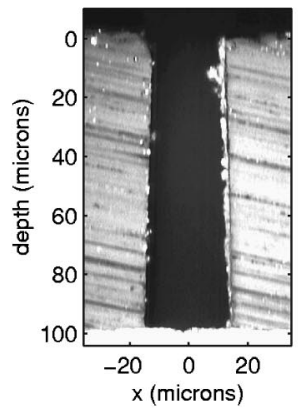

(c)
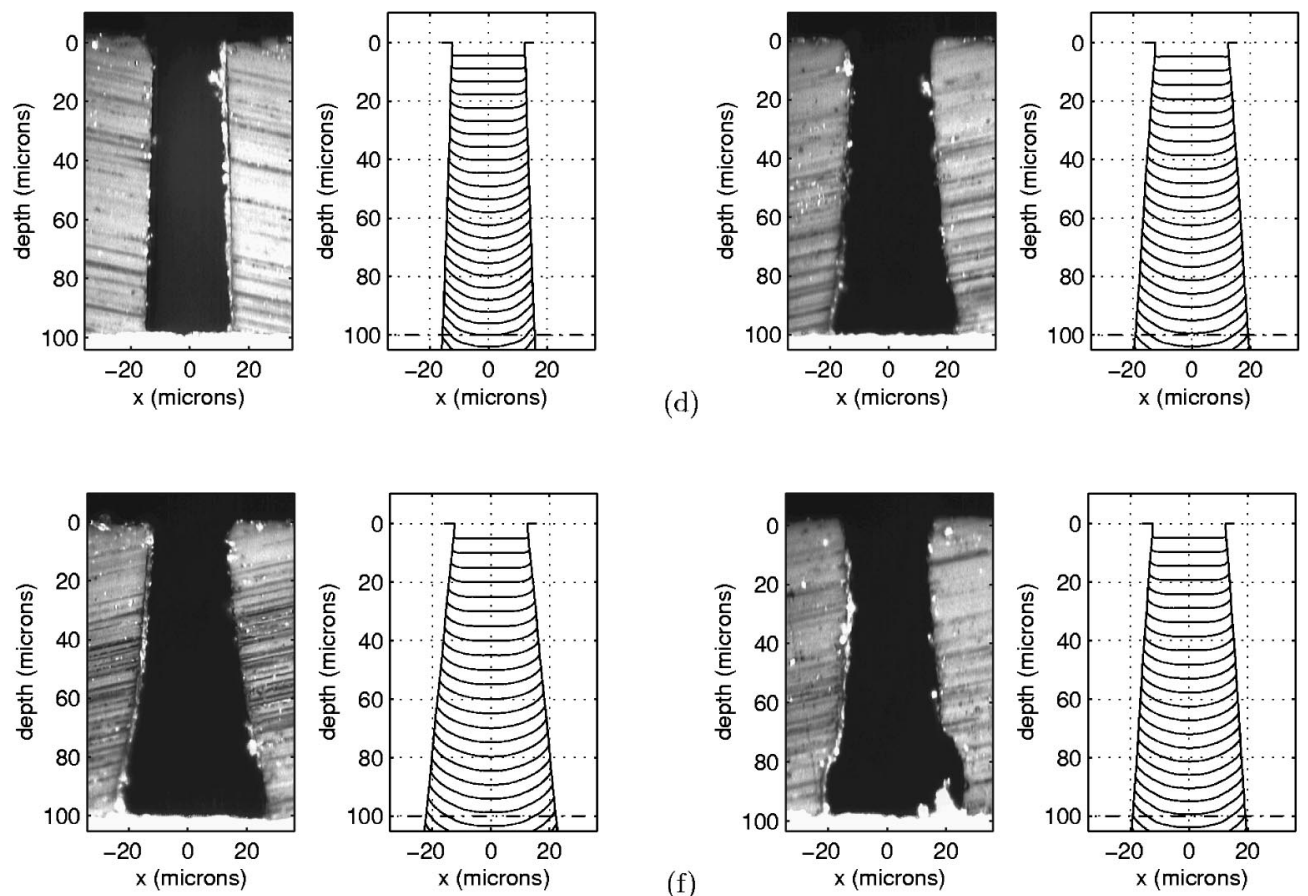

(d)

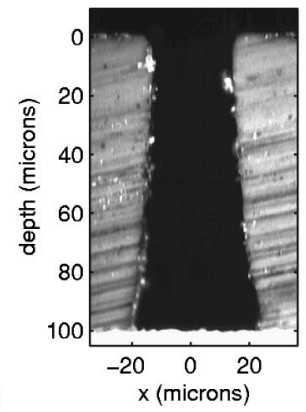

(b)

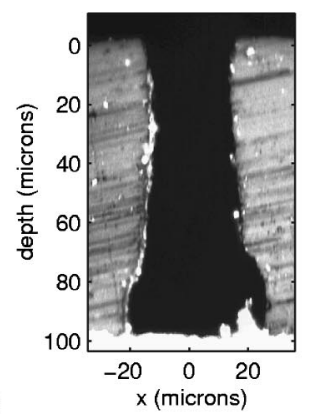

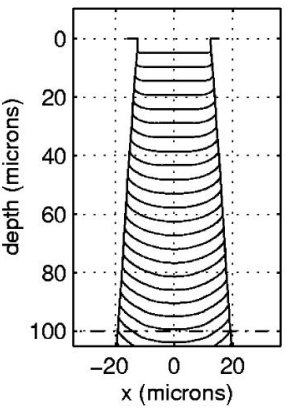

FIG. 6. Comparison between profiles predicted by our model (right) and actual ablated structures (left) for various fluences $E$ : (a) $E=0.15 \mathrm{~J} \mathrm{~cm}^{-2}, N$ $=600$; (b) $E=0.25 \mathrm{~J} \mathrm{~cm}^{-2}, N=600$; (c) $E=0.5 \mathrm{~J} \mathrm{~cm}^{-2}, N=600$; (d) $E=0.75 \mathrm{~J} \mathrm{~cm}^{-2}, N=600$; (e) $E=1.0 \mathrm{~J} \mathrm{~cm}^{-2}, N=600 ; \quad(\mathrm{f}) E=0.75 \mathrm{~J} \mathrm{~cm}{ }^{-2}$, $N=1000$ - overetched, showing flaring.

a wavelength of $193 \mathrm{~nm}$ with a nominal pulse width of $17 \mathrm{~ns}$. Trenches were formed by imaging rectangular apertures through a $15 \times, 0.28 \mathrm{NA}$ reflecting objective. The illumination was provided by a $6 \times 6$ fly's eye homogenizer with an effective NA in the image space of 0.2 . The apertures were machined in brass foil using a Nd:YAG laser, and were designed to produce $200 \mu \mathrm{m}$ long trenches with nominal widths of $25 \mu \mathrm{m}$ at the top surface of the resist.

The etch rate per pulse for normally incident uniform radiation was derived by fitting an empirical function to experimental data, obtained by measuring (with a DEKTAK profilometer) the surface profiles of samples exposed to a small number of pulses. The following empirical function was found to fit the etch rate data well

$$
h(f)=d \tan ^{-1}\left[a \ln \left(\frac{f}{f_{0}}\right)\right],
$$

with the values $a=0.82, f_{0}=0.066 \mathrm{~J} \mathrm{~cm}^{-2}$, and $d$ $=0.22 \mu \mathrm{m}$, where $f_{0}$ corresponds to an ablation threshold. Figure 4 shows the experimental data and the fitted empirical function.
A number of trenches were then produced for different fluences and for different numbers of laser pulses. These were sectioned using a wafer dicing saw, and photographed under an optical microscope. Figures 5 and 6 show a representative selection of profiles predicted by the model, together with cross sections of ablated structures machined under the same conditions: Fig. 5 for different numbers of pulses and Figs. 6(a)-6(e) for different fluences. The predicted profiles in Fig. 6 show the evolution of the trench shape at 20-pulse intervals for different fluences in addition to the final prediction. Agreement between the final profiles predicted by the model and the actual structures is very good, except in Figs. 6(a) and 6(e), which are for the lowest and highest fluences. Significantly, the model correctly predicts tapering of the trenches towards the bottom at fluences of 0.15 and $0.25 \mathrm{~J} / \mathrm{cm}^{2}$, and widening at higher fluences. Figure 6(f) shows the effect of a large number of pulses with high fluence. Note the flaring at the bottom of the trench which is probably due to back reflections from the substrate. This effect, which is also present to a lesser extent in Fig. 6(e), is not accounted for in the modeling. 


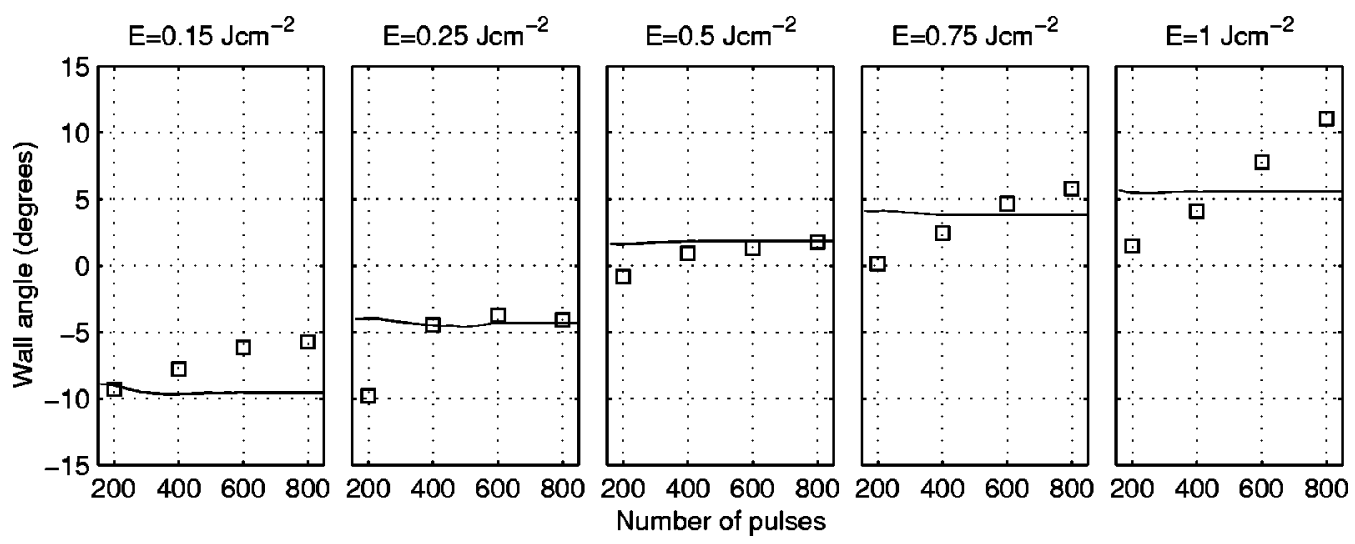

FIG. 7. Variation of wall angle with number of pulses at different fluences for Du Pont RISTON. Solid lines are model predictions and points are experimental data.

In Fig. 7, the predicted variations of wall angle with exposure dose at different fluences are compared with experimental data. Trenches were machined on a wafer which was then diced and photographed to produce images of the trench cross sections. Each data point corresponds to the wall angle for a separate trench which is taken (arbitrarily) as the angle between the normal to the substrate surface and a leastsquares linear fit to points on the sidewall (ten data points for each sidewall, with depths evenly distributed between $10 \%$ and $90 \%$ of the trench depth). In most cases the sidewall was not perfectly straight and so a straight line fit could not fully describe the profile. However, it did provide an unbiased measure of the average wall angle, allowing a simple quantitative comparison between the model and the experimental data. Note that the model predictions in Fig. 7 are also derived from best fit straight lines of the final trench ablated with the given number of pulses. A positive angle indicates the trench widens towards the bottom.

There is agreement to within a few degrees between the experiment and the modeling predictions except for the shallowest trenches (for 200 pulses) and for trenches machined at very low and very high fluences. At the higher fluences, the flaring at the bottom of the trenches, which increases with number of pulses, is largely responsible for the increase of the measured wall angle with number of pulses. Nevertheless, there does appear to be a slight increase of wall angle with the number of pulses for all the fluences, which is not predicted by the model.

\section{CONCLUSIONS}

A new numerical model for the excimer laser ablation of microstructures has been presented which is based on propagating the surface of the structure pulse-by-pulse. The model uses the full three-dimensional aerial image in the region of the structure, taking into account the partial coherence properties of the illumination typical in excimer laser micromachining setups. This is combined with a model for the etch rate for non-normally incident radiation to calculate the local etch rate over the surface for each laser pulse. A large number of assumptions have been made in deriving the model, in particular with regard to how the developing structure itself affects the propagation of the light in the aerial image and in the behavior of the ablation etch rate with the incident radiation. Despite these approximations, the model has been shown to be in good agreement with experimental results for the ablation of two-dimensional trench structures using a commercial micromachining workstation. The model shows good agreement for trenches that are significantly deeper than the depth of focus of the image $(\sim 5 \mu \mathrm{m})$ and machined at moderate values of fluence. The results indicate that a simple explanation for the ablation of deep (c.f. the depth of focus) structures is possible based solely on the properties of the aerial image. To date we have obtained experimental data only for RISTON dry film photoresist. Results for more common materials, in particular PMMA and polyimide, will be published in a later paper.

A possible explanation for the discrepancies in the wall angle predictions at the lower fluences is the crude nature of the etch rate model used, this being purely empirical, derived from experimental data and assumptions about its variation with angle of incidence. The slope of the empirical function describing the etch rate is greatest at low fluences near the ablation threshold, so inaccuracies such as variations in the total fluence between pulses (which have not been included in the model), will be most important here. It is most likely that the crude nature of the etch rate formula is the dominant factor limiting the applicability of the model in the low fluence regime. Neglect of reflected contributions may also be relevant. The discrepancies at high fluence, in particular the flaring at the bottom of actual ablated structures, probably result from substrate reflections. This flaring does not appear to happen at the lower fluences. There is a simple explanation for this based on geometrical arguments. Reflections off the substrate serve to reduce the angle of incidence of the reflected waves onto undercut sidewalls, while increasing it for in-tapering sidewalls. This increases the ablative effect of reflected waves on the former while reducing it on the latter. Consequently, it is expected that ablation due to reflections from the bottom of the substrate will be far greater for undercut than for in-tapering sidewalls.

\section{ACKNOWLEDGMENTS}

This work was funded by the U.K. Engineering and Physical Sciences Research Council (Grant No. GR/ 
K71479). The experimental data were obtained at the Institute of Microtechnology in Mainz (IMM), under the Large Scale Facility Scheme of the EC Human Capital and Mobility Program (Contract No. ERBCHGECT930052). The authors are grateful to Exitech Ltd for providing technical data on the Series 7000 micromachining workstation.

${ }^{1}$ Y. Kawamura, K. Toyoda, and S. Namba, Appl. Phys. Lett. 40, 374 (1982).

${ }^{2}$ R. Srinivasan and V. Maynebanton, Appl. Phys. Lett. 41, 576 (1982).

${ }^{3}$ F. N. Goodall, R. A. Lawes, and G. G. Arthur, Microelectron. Eng. 11, 187 (1990).

${ }^{4}$ H. J. Kahlert, B. Sarbach, B. Burghardt, and B. Klimt, Proc. SPIE 1835, 110 (1992).

${ }^{5}$ J. R. Lankard and G. Wolbold, Appl. Phys. A: Solids Surf. 54, 355 (1992). ${ }^{6}$ F. Bachmann, Chemtronics 4, 149 (1989).

${ }^{7}$ M. C. Gower, in Laser Processing in Manufacturing, edited by R. C. Crafer and P. J. Oakley (Chapman \& Hall, London, 1993), pp. 189-261.

${ }^{8}$ S. L. Trokel, R. Srinivasan, and B. Braren, Am. J. Ophthalmol. 96, 710 (1983).

${ }^{9}$ D. L. Singleton, G. Paraskevopoulos, G. S. Jolly, R. S. Irwin, D. J. McKenney, W. S. Nip, E. M. Farrell, and L. A. J. Higginson, Appl. Phys. Lett. 48, 878 (1986).

${ }^{10}$ H. Ota, T. Oda, and M. Kobayashi, Proceedings of the 1995 IEEE MEMS Workshop, pp. 197-202.

${ }^{11}$ R. Pethig, J. P. H. Burt, A. Parton, N. Rizvi, M. S. Talary, and J. A. Tame, J. Micromech. Microeng. 8, 57 (1998).

${ }^{12}$ J. Arnold, U. Dasbach, W. Ehrfeld, K. Hesch, and H. Lowe, Appl. Surf. Sci. 86, 251 (1995).

${ }^{13}$ R. A. Lawes, A. S. Holmes, and F. N. Goodall, Microsystem Technol. 3, 17 (1996).

${ }^{14}$ B. J. Garrison and R. Srinivasan, J. Appl. Phys. 57, 2909 (1985).
${ }^{15}$ J. E. Andrew, P. E. Dyer, D. Forster, and P. H. Key, Appl. Phys. Lett. 43, 717 (1983).

${ }^{16}$ H. H. G. Jellinek and R. Srinivasan, J. Phys. Chem. 88, 3048 (1984).

${ }^{17}$ E. Sutcliffe and R. Srinivasan, J. Appl. Phys. 60, 3315 (1986).

${ }^{18}$ S. Küper and M. Stuke, Appl. Phys. B: Photophys. Laser Chem. 44, 199 (1987).

${ }^{19}$ S. R. Cain, F. C. Burns, and C. E. Otis, J. Appl. Phys. 71, 4107 (1992).

${ }^{20}$ S. R. Cain, F. C. Burns, C. E. Otis, and B. Braren, J. Appl. Phys. 72, 5172 (1992).

${ }^{21}$ G. H. Pettit and R. Sauerbrey, Appl. Phys. A: Solids Surf. 56, 51 (1993).

${ }^{22}$ S. Küper, J. Brannon, and K. Brannon, Appl. Phys. A: Solids Surf. 56, 43 (1993)

${ }^{23}$ G. C. Dcouto and S. V. Babu, J. Appl. Phys. 76, 3052 (1994).

${ }^{24}$ R. Srinivasan, in Laser Ablation, edited by J. C. Miller (Springer, Berlin, 1994), pp. 107-133.

${ }^{25}$ P. E. Dyer, S. D. Jenkins, and J. Sidhu, Appl. Phys. Lett. 49, 453 (1986).

${ }^{26}$ P. E. Dyer, D. M. Karnakis, P. H. Key, and P. Monk, Appl. Surf. Sci. 96-8, 415 (1996).

${ }^{27}$ T. W. Hodapp and P. R. Fleming, J. Appl. Phys. 84, 577 (1998).

${ }^{28}$ A. Ishikawa, M. Izutsu, and T. Sueta, Appl. Opt. 29, 5064 (1990).

${ }^{29}$ K. Hirayama, E. N. Glytsis, and T. K. Gaylord, J. Opt. Soc. Am. A 14, 907 (1997).

${ }^{30} \mathrm{~J}$. W. Goodman, Introduction to Fourier Optics (McGraw-Hill, New York, 1968)

${ }^{31}$ K. A. Valiev, L. V. Velikov, G. S. Volkov, and D. Y. Zaroslov, J. Vac. Sci. Technol. B 7, 1616 (1989).

${ }^{32}$ M. Born and E. Wolf, Principles of Optics, 6th ed. (Pergamon, Oxford, UK, 1980).

${ }^{33}$ H. Lorenz, L. Paratte, R. Luthier, N. F. de Rooij, and P. Renaud, Sens. Actuators A53, 364 (1996).

${ }^{34}$ J. Zhu, A. S. Holmes, J. Arnold, R. A. Lawes, and P. D. Prewett, Microelectron. Eng. 30, 365 (1996). 